\title{
Excitonic effects in quantum wires
}

\author{
Guido Goldoni, Fausto Rossi, and Elisa Molinari \\ Istituto Nazionale Fisica della Materia (INFM) and \\ Dipartimento di Fisica, Università di Modena \\ via Campi 213/A, I-41100 Modena, Italy
}

(July 1, 2021)

\begin{abstract}
We review the effects of Coulomb correlation on the linear and non-linear optical properties of semiconductor quantum wires, with emphasis on recent results for the bound excitonic states. Our theoretical approach is based on generalized semiconductor Bloch equations, and allows full three-dimensional multisubband description of electron-hole correlation for arbitrary confinement profiles. In particular, we consider $\mathrm{V}$ - and T-shaped structures for which significant experimental advances were obtained recently. Above band gap, a very general result obtained by this approach is that electron-hole Coulomb correlation removes the inverse-square-root single-particle singularity in the optical spectra at band edge, in agreement with previous reports from purely one-dimensional models. Strong correlation effects on transitions in the continuum are found to persist also at high densities of photoexcited carriers.

Below bandgap, we find that the same potential- (Coulomb) to kinetic-energy ratio holds for quite different wire cross sections and compositions. As a consequence, we identify a shape- and barrier-independent parameter that governs a universal scaling law for exciton binding energy with size. Previous indications that the shape of the wire cross-section may have important effects on exciton binding are discussed in the light of the present results.
\end{abstract}

Classification scheme: 78.66.F, 73.20.D

\section{INTRODUCTION}

Much of the interest in the optical properties of quantum wires was initially motivated in terms of their singleparticle properties: The well known van Hove divergence in the one-dimensional (1D) density-of-states (DOS) was expected to induce sharp peaks in the optical spectra of quantum wires, thereby allowing to achieve structures with improved optical efficiency as compared to their two-dimensional (2D) and three-dimensional (3D) counterpartsl.

¿From the theoretical point of view, the importance of Coulomb correlation in determining the optical response of quantum wires was pointed out by Ogawa and Takagaharat. By solving a purely 1D Schrödinger equation in terms of a modified Coulomb potential, they found that the $1 / \sqrt{E}$ singularity at the band edge was smoothed when electron-hole interaction was taken into account. For a few years, however, the impact of such result was very limited. The reason is probably twofold. On the one hand, an idealized 1D model was thought to be far from the physics of the (wide) quantum wires that could be actually fabricated. On the other hand, it was expected that at high carrier densities - the regime of interest for most applications - the effects of electronhole interactions above band gap would be washed out, in analogy with the behaviour of bound excitonic states below band gap. From an experimental point of view, "sharp" 1D features at the band edge are generally not observed in the optical spectra of quantum wires; however, owing to disorder-induced inhomogeneous broadening, it is hard to single out the role played by electron- hole correlation in their suppression.

An additional effort was therefore needed on the theoretical side, in order to assess the effects of Coulomb interaction in the quantum wires that are made available by the present technology. These include structures obtained by epitaxial growth on non-planar substrates (Vshaped wires) 13 growth (T-shaped wires) 8: here, the lateral extension of the ground single-particle states is still significant (see e.g. Fig. 1), the excited states gradually approach a 2Dlike behaviour, and the subband separation is relatively small, so that the coupling between different subbands may be important.

For this reason, we have recently developed 10 a fully $3 \mathrm{D}$ approach that allows the analysis of Coulomb correlation in realistic quantum wires, and is based on multisubband semiconductor Bloch equations (SBE)11. A first set of studies 9 focused precisely on the effect of electronhole coupling on the optical spectra above the onset of the continuum. The main results can be summarized as follows: (i) The correlated absorption spectra of realistic wires do show a strong quenching of the 1D singleparticle singularity, arising from the fact that the oscillator strength vanishes when the excess energy decreases to approach the band edge; this effect does not depend on details of the wire cross section. The Sommerfeld factor, which is greater than unity in the bulk and in quantum wells (the so-called Coulomb enhancement), is instead smaller than unity in quantum wires (Coulomb suppression), thus reducing the influence of dimensionality on the optical spectra. In this respect, the findings of Ref. 2 are therefore confirmed and their validity is extended to 
realistic quantum wires with finite cross-section and arbitrary number of subbands. (ii) The Coulomb-induced suppression of the 1D singularity is found to hold not only in the linear regime but also at higher carrier densities, and to persist in the gain regime.

The above results call for a discussion of their possible implications for perspective devices, since the initial motivations - based on single particle models - are now recognized to be far too simplified. At the same time, the theoretical approach that was developed in Ref. 9 can now be extended and used for an accurate study of correlation effects below band-gap, i.e. for predicting the properties of bound excitonic states in realistic wires 10 . Beside their fundamental interest, these properties are of relevance for possible applications in devices (e.g. nonlinear optical switches) that would benefit of enhanced exciton binding energies.

The rest of the paper is organized as follows. In Section II we briefly summarize the state of the art in the theoretical study of bound excitons in quantum wires, and recall the key features of our approach. In Section III we summarize and discuss our main results for T-shaped and V-shaped quantum wires and compare critically with experimental data.

\section{BOUND EXCITONS IN QUANTUM WIRES: THEORETICAL BACKGROUND}

In the pure 1D limit, we know from the solution of the $1 \mathrm{D}$ hydrogenic problem 12 that the exciton binding energy diverges. In real systems, where the cross section of the wire is finite, unphysical 1D divergencies disappear because the Coulomb interaction has a $3 \mathrm{D}$ nature. The resulting exciton binding energy is finite, and its value depends on the confining potential which determines the spatial extension of the interacting electron and hole wavefunctions.

Most of the early models for excitons in quantum wires did not rely on a 3D description of the system: in such cases, divergencies are avoided by assuming effective 1D potentials (usually Coulomb-like potentials regularized at the origin) to simulate the effect of the lateral extension of the wire. While some of these calculations have led to the understanding of important features of forrelated spectra 2,13 - later confirmed by $3 \mathrm{D}$ studies 9 , they are intrinsically unable to account for the dependence of binding energies on the specific confining potential. Three-dimensional studies of Coulomb interactions in wires were also developed, mostly based on variational approaches for the wavefunctions 1416.

With the adrent of optical studies on V-shaped and T-shaped wirest 3 , 8 , several groups have realized the importance of the specific confining potential in determining single-particle properties of electrons and holes in the wires. Results based on different approximations are reported e.g. in Refs. 4, 5, 17, 18 and 1822 for V- and T-like geometries, respectively.
It was still unclear, however, whether the shape of the wire cross section could influence significantly the exciton binding energies, $E_{b}$. More generally, the dependence of $E_{b}$ on the parameters defining the confining potential, and its scaling with size were still highly controversial. Theoretical predictions obtained through variational calculations for model wire geometries indicate that the scaling of $E_{b}$ should be simply governed by the extension of the single-particle wavefunctions in the plane perpendicular to the free wire direction, and much less sensitive to the shape of the wire 14.23 .24$.

In view of the current debate on experimental values of $E_{b}$ and their interpretation, it is useful to reexamine the influence of the wire cross section through the SBE approach, that involves no assumption on the form of the variational wavefuntions. The implementation of the generalized SBE method for quantum wires is described in full detail in Ref. 9. Here, we just stress once more that this approach fully describes the 3D nature of the electron-hole Coulomb interaction. Moreover, our multisubband approach includes on the same footing both confined and continuum states; this is especially important in order to properly describe T-wires, where the $1 \mathrm{D}$ ground state is relatively close to the $2 \mathrm{D}$ continua of the parent QWs.

Note also that, in addition to quantum-confinement effects, the method based on SBEs can be generalized to include dielectric-confinement effects, i.e., the contribution due to the dielectric-constant mismatch at the various material interfaces ] . Contrary to some higher-symmetry systems, where these effects can be incorporated through simple image-charge methods, for V-and T-shape geometries their calculation require a full $3 \mathrm{D}$ analysis. Within our scheme, such 3D solution is performed in the same plane-wave representation used for the solution of the single-particle Schrödinger equation. The renormalized electron-hole Coulomb potential -obtained as the solution of the Poisson equation with a spatially varying dielectric constant - is then used to compute the optical response through the polarization equation [eq. (17) in Ref. 9]. The exciton binding energy obtained in this way reflects both quantum- and dielectric-confinement effects.

A more detailed account of the numerical approach for solving coupled SBE-Poisson equations will be given in a forthcoming publication. Their solution is obtained by direct numerical evaluation of the polarization eigenvalues and eigenvectors, which fully determine the absorption spectrum. The main ingredients are the singleparticle energies and wavefunctions, which in turn are numerically computed (using a plane-wave expansion) starting from the real shape of the 2P confinement potential deduced from TEM, as in Ref.t (for an example see Fig. 1).

In the next Section, the results for realistic wires will be discussed in terms of the exciton binding energy $E_{b}$ (calculated as the difference between the ground-state energy of the correlated and uncorrelated Hamiltonians), 
and the ground-state average of the Coulomb and the kinetic energy, $\langle V\rangle$ and $\langle K\rangle$. The latter is defined as the difference $\langle K\rangle=E_{b}-\langle V\rangle$. Therefore, the ratio $\alpha=-\langle V\rangle /\langle K\rangle$ is the equivalent of a virial coefficient: in a purely $3 \mathrm{D}$ or $2 \mathrm{D}$ system, $\alpha=2$ as a direct consequence of the virial theorem. In the presence of an additional confining potential, the virial theorem no longer holds and $\alpha$ may deviate from 2 . It is particularly interesting to verify whether values of $\alpha>2$ can be achieved in realistic quantum wires, as this would imply enhanced binding energies with respect to the extreme 2D limit.

\section{RESULTS FOR V- AND T-SHAPED QUANTUM WIRES}

We have considered the typical V-and T-confinement profiles illustrated in the insets of Fig. 2, and scaled them by varying the corresponding geometrical parameters $\left(L_{V}\right.$ and $\left.L_{T}\right)$ in a wide range of values. For each value of these parameters, we have considered two sets of conduction and valence band offsets, simulating both low- $x \mathrm{Al}_{x} \mathrm{Ga}_{1-x} \mathrm{As}$ and pure AlAs barrier compositions (samples labeled "1" and "2", respectively).

The calculated binding energies are illustrated by thick solid lines in Fig. 2. As expected, they increase with decreasing $L_{V}$ or $L_{T}$; for samples " 2 ", corresponding to AlAs barriers, the excitonic binding is larger than in the case of low-barrier samples " 1 ". Note the different scale of both panels: a same value of $E_{b}$ can be obtained in a $\mathrm{V}$ - or T-shaped wire; to this purpose, the geometrical parameters must be such that $L_{T}<L_{V}$. For an example of the corresponding single-particle wavefunctions, see the plots in Fig. 1 which refer to the lowest circled values of $E_{b}$ in each panel.

The thinner lines in Fig. 2 represent the average potential energy, $\langle V\rangle$, for the same sets of samples. The contribution of $\langle V\rangle$ appears to dominate over $\langle K\rangle$, and to determine the $E_{b}$ dependence on the geometrical parameters. It is therefore useful to define the quantity:

$$
a=\left\langle\frac{1}{\mathbf{r}}\right\rangle^{-1},
$$

where the average is performed over the ground state. For a 3D bulk semiconductor, $a$ coincides with the usual exciton Bohr radius $a_{\circ}$, and can thus be considered as an effective Bohr radius in the wire. The insets in Fig. 2 show $a$ as a function of the relevant geometrical parameter, $L_{V}$ or $L_{T}$. A same value of a corresponds to different values of $L_{V}$ and $L_{T}$, with $L_{V}$ always larger than $L_{T}$. Note that samples with similar binding energies correspond to similar values of $a$ (see again the circled points in Fig. 2).

Finally, by plotting the binding energy $E_{b}$ of all samples vs the corresponding exciton radius $a$ (Fig. 3), we obtain a universal (shape and barrier independent) curve, $E_{b} \sim \frac{1}{a}$. A universal scaling of the mean potential and kinetic energy is apparent in the $\langle V\rangle$ vs $\langle K\rangle$ plot reported in the inset of Fig. 3; to a very good approximation, all sets of points for $\mathrm{V}$ - and $\mathrm{T}$-wires fall on a straight line with slope $\alpha$ very close to 4 . For comparison, we have performed analogous calculations for a set of QWs: we find that $E_{b}$ scales with $a$ similarly to wire structures, although with a different prefactor, corresponding approximately to $\alpha=2$ (inset of Fig. 3).

The above findings confirm that quantum wire confinement is indeed advantageous for the purpose of obtaining enhanced exciton binding, and provide a general and quantitative prescription for tailoring $E_{b}$ by tuning the effective exciton Bohr radius $a$ through the geometrical size parameters. At the same time, they clearly indicate that only minor effects can be introduced by tailoring the shape of the wire cross-section, unless smaller values of the effective Bohr radius $a$ are obtained. This last conclusion is in apparent contradiction with some findings that have beep reported for T-wires d. A reinterpretation of these data 25 seems now to reconcile them with the present theoretical picture, as well as with the previous experimental resultst 26 .

Before concluding, we briefly comment on the role of dielectric confinement in these systems. In agree ment with previous investigations in quantum wells 27, our analysis confirms that for GaAs/AlGaAs-based structures dielectric confinement has a minor influence as compared to quantum confinement. A slightly larger effect, still not more than a few $\mathrm{meV}$, is found for the case of the GaAs/AlAs T-shaped structures; for example, for a symmetric GaAs/AlAs T-wire with $L_{T}=5.4 \mathrm{~nm}$ (sample $\mathrm{S} 2$ in Ref. (7), we found a dielectric-confinement induced enhancement of $\sim 3 \mathrm{meV}$. This increase is not attributed to the particular shape of the wire, but to the larger dielectric mismatch of the GaAs/AlAs interface and to the smaller geometrical size of the wire.

In summary, we have presented a theoretical analysis of the optical properties of realistic quantum wires, based on a numerical solution of the semiconductor Bloch equations describing the multisubband 1D system. We have applied such approach to typical T- and V-shaped structures, whose parameters reflect the current state-of-theart in quantum-wire fabrication. The calculations show that exciton binding energies in wires may indeed reach values much larger than in quantum wells; the value of the coefficient $\alpha \sim 4$, that is found to hold in a wide range of geometrical wire parameters, allows binding energies larger than the ideal 2D limit. The scaling of the exciton binding energy is governed by a universal parameter that limits the possible differences due to variations in the shape of the wire cross-section. Our results for realistic $\mathrm{V}$ - and $\mathrm{T}$-wires in the strong confinement regime are consistent with the available experimental data and offer a guideline for tailoring binding energies in these structures.

Acknowledgements. We are grateful to H. Sakaki for useful discussions, and to T.L. Reinecke for communicating results prior to publication. 
${ }^{1}$ For a review see e.g. R. Cingolani and R. Rinaldi, Rivista Nuovo Cimento 16, 1 (1993).

2 Tetsuo Ogawa and Toshihide Takagahara, Phys. Rev. B43, 14325 (1991); ibid. B44, 8138 (1991).

${ }^{3}$ E. Kapon et al., Phys. Rev. Lett. 63, 430 (1989).

${ }^{4}$ R. Rinaldi et al., Phys. Rev. Lett. 73, 2899 (1994).

${ }^{5}$ M. Grundmann et al., J. Nonlin. Optical Phys. and Materials 4, 99 (1995), and references therein.

${ }^{6}$ L. Pfeiffer et al., Appl. Phys. Lett. 56, 967 (1990); W. Wegscheider et al., Appl. Phys. Lett. 65, 2510 (1994).

7 T. Someya, H. Akiyama, and H. Sakaki, Phys. Rev. Lett. 76, 2965 (1996).

${ }^{8}$ H. Gislason, W. Langbein, J.M. Hvam, Appl. Phys. Lett. 69, 3248 (1996).

${ }^{9}$ F. Rossi and E. Molinari, Phys. Rev. Lett. 76, 3642 (1996); Phys. Rev. B53, 16462 (1996).

${ }^{10}$ F. Rossi, G. Goldoni, and E. Molinari, Phys. Rev. Lett. 78, 3527 (1997).

${ }^{11}$ See e.g. J. Shah, Ultrafast Spectroscopy of Semiconductors and Semiconductor Nanostructures (Springer, Berlin, 1996); H. Haug and S.W. Koch, Quantum Theory of the Optical and Electronic Properties of Semiconductors, 3rd Edn., World Scientific, Singapore (1994).

${ }^{12}$ R. Loudon, Am. J. Phys. 27, 649 (1959); R.J. Elliot and R. Loudon, J. Phys. Chem. Solids 8, 382 (1959); 15, 196 (1960).

${ }^{13}$ H. Ando, H. Oohashi, H. Kanbe, J. Appl. Phys. 70, 7024 (1991).

14 M.H. Degani and O. Hipolito, Phys. Rev. B35, 9345 (1987).

15 J.W. Brown and H.N. Spector, Phys. Rev. B35, 3009 (1987).

16 Y.C. Chang, L.L. Chang, and L. Esaki, Appl. Phys. Lett. 47, 1324 (1985).

${ }^{17}$ D.S. Citrin and Y.-C. Chang, IEEE J. Quantum Electronics 29, 97 (1993).

${ }^{18}$ G. Goldoni et al., App. Phys. Lett. 69, 2965 (1996); G. Goldoni, F. Rossi, E. Molinari, A. Fasolino, Phys. Rev. B55, 7110 (1997).
${ }^{19}$ D. Gershoni et al., Phys. Rev. Lett. 65, 1631 (1990).

${ }^{20}$ A.A. Kiselev and U. Rossler, Semicond. Sci. Technol. 11, 203 (1996).

${ }^{21}$ W. Langbein, H. Gislason, J.M. Hvam, Phys. Rev. B54, 14595 (1996).

${ }^{22}$ D. Brinkmann, A. Löffler, and G. Fishman, Il Nuovo Cimento 17, 1389 (1995).

${ }^{23}$ P. Lefebvre, P. Christol, H. Mathieu, and S. Glutsch, Phys. Rev. B52, 5756 (1995), and references therein.

${ }^{24}$ S.N. Walck, T.L. Reinecke, and P.A. Knipp, Phys. Rev. B (1997), in press.

${ }^{25} \mathrm{H}$. Sakaki, this conference.

${ }^{26}$ H. Weman et al., Phys. Rev. B53, R6959 (1996), and references therein.

${ }^{27}$ L.C. Andreani and A. Pasquarello, Phys. Rev. B42, 8928 (1996).

FIG. 1. Single particle electron envelope functions for a $\mathrm{V}$-wire with $L_{V}=8.7 \mathrm{~nm}$ and conduction band offset of 150 meV (left panel), and a T-wire with $L_{T}=5.4 \mathrm{~nm}$ conduction band offset of $243 \mathrm{meV}$ (right panel). The exciton binding energies of these samples are the lower circled dots in Fig. 2.

FIG. 2. Exciton binding energy $E_{b}$ and mean potential energy $\langle V\rangle$ for a set of $\mathrm{V}$-wires (left) and T-wires (right). Full dots indicate samples with pure AlAs barriers, while empty dots indicate low-Al content samples; complete sample parameters are summarized in Table 1 of Ref. 10. In each panel, the left insets shows the wire geometry, with indication of the relevant geometrical parameter; the right insets shows the calculated effective exciton Bohr radius, $a$, vs the relevant geometrical parameter. The cifcled points refer to sample parameters corresponding to Ref 4 (V-wires) and Ref.U (T-wires).

FIG. 3. Exciton binding energy, $E_{b}$, vs effective exciton Bohr radius, $a$, for $\mathrm{V}$ - and $\mathrm{T}$-wires, and for a set of QWs. Dashed curves are a fitting to $1 / a$. The inset shows the average potential vs kinetic energy, falling on a straight line with slope $\alpha \simeq 4$ for all wire samples. Results for QW structures are also shown for comparison; in this case $\alpha \simeq 2$. Solid lines are a linear fit to the calculated points. 

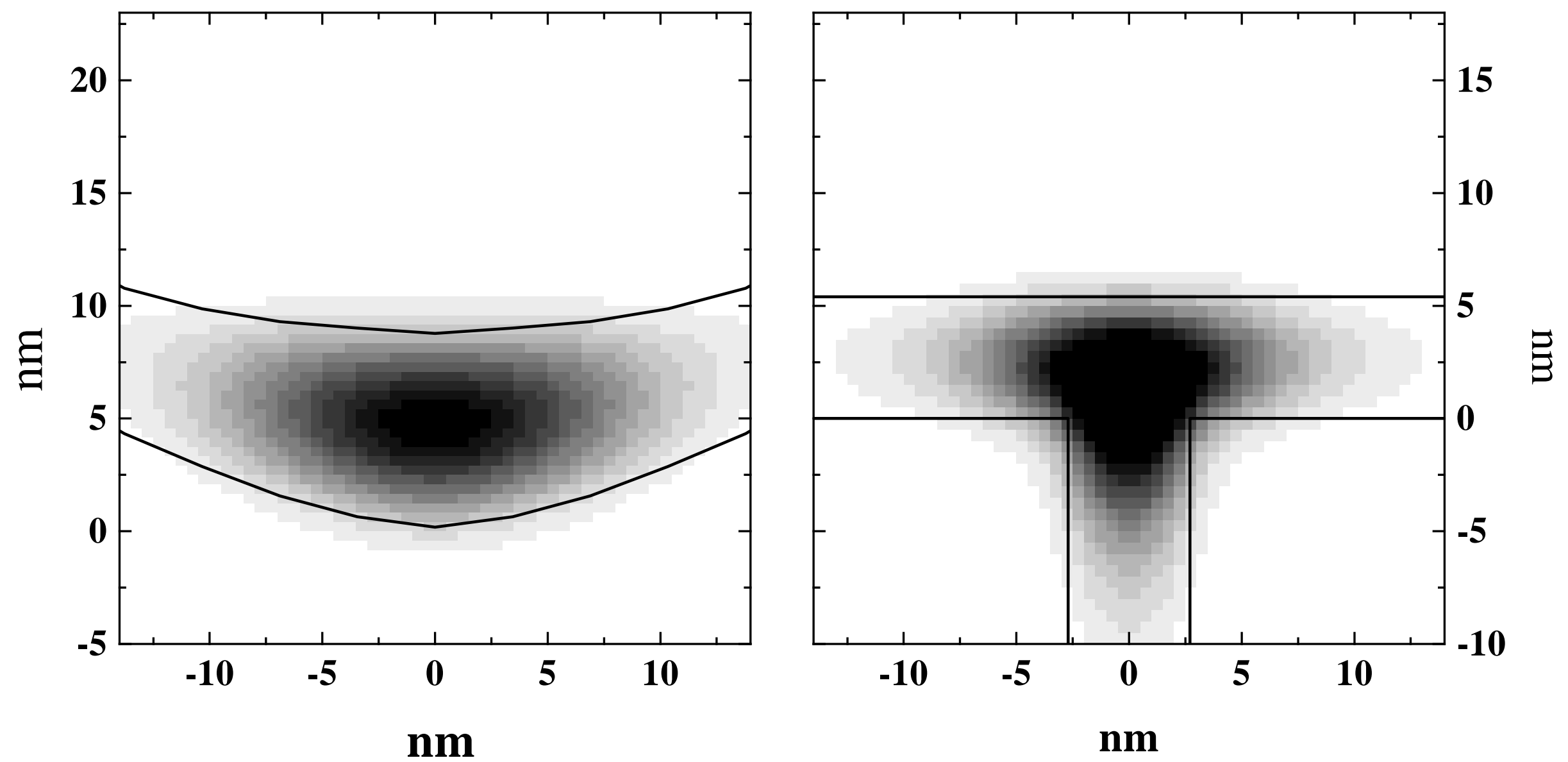

G. Goldoni et al. - Fig. 1 

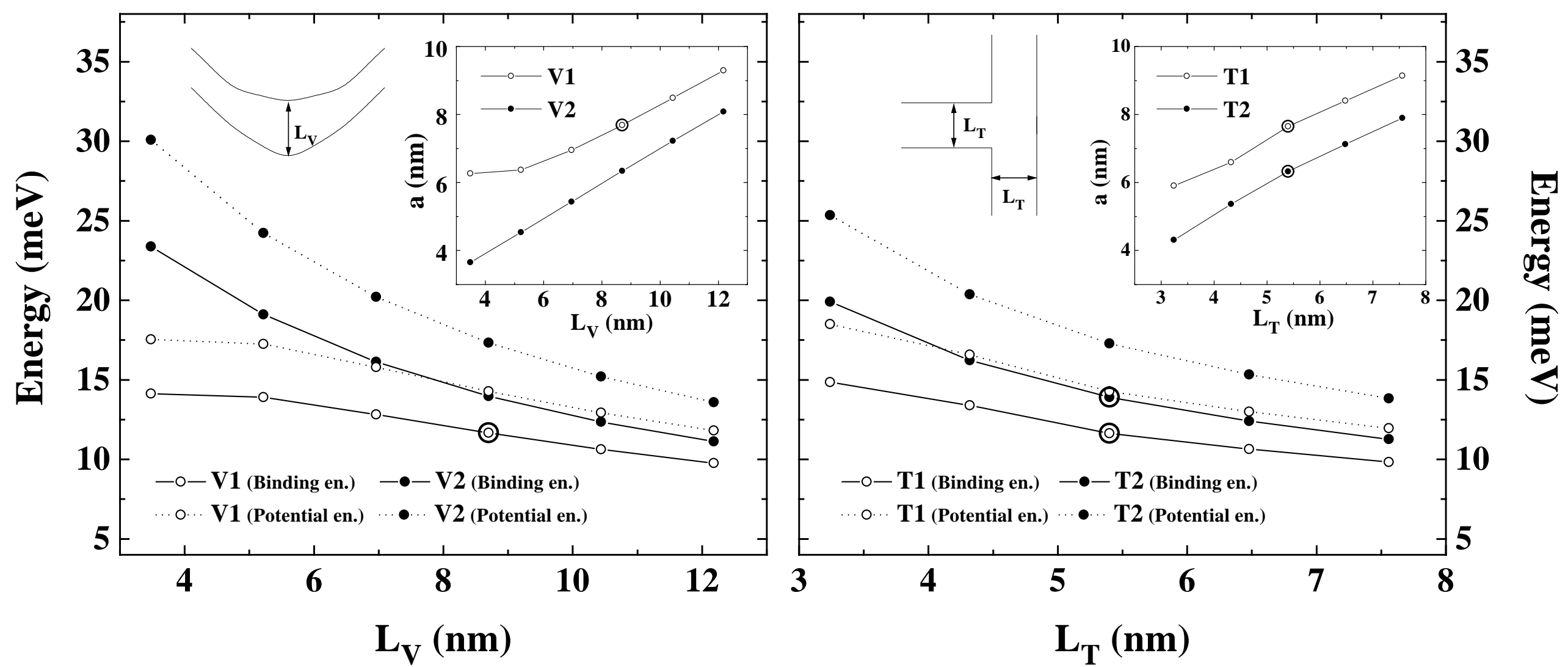

G. Goldoni et al. - Fig. 2 


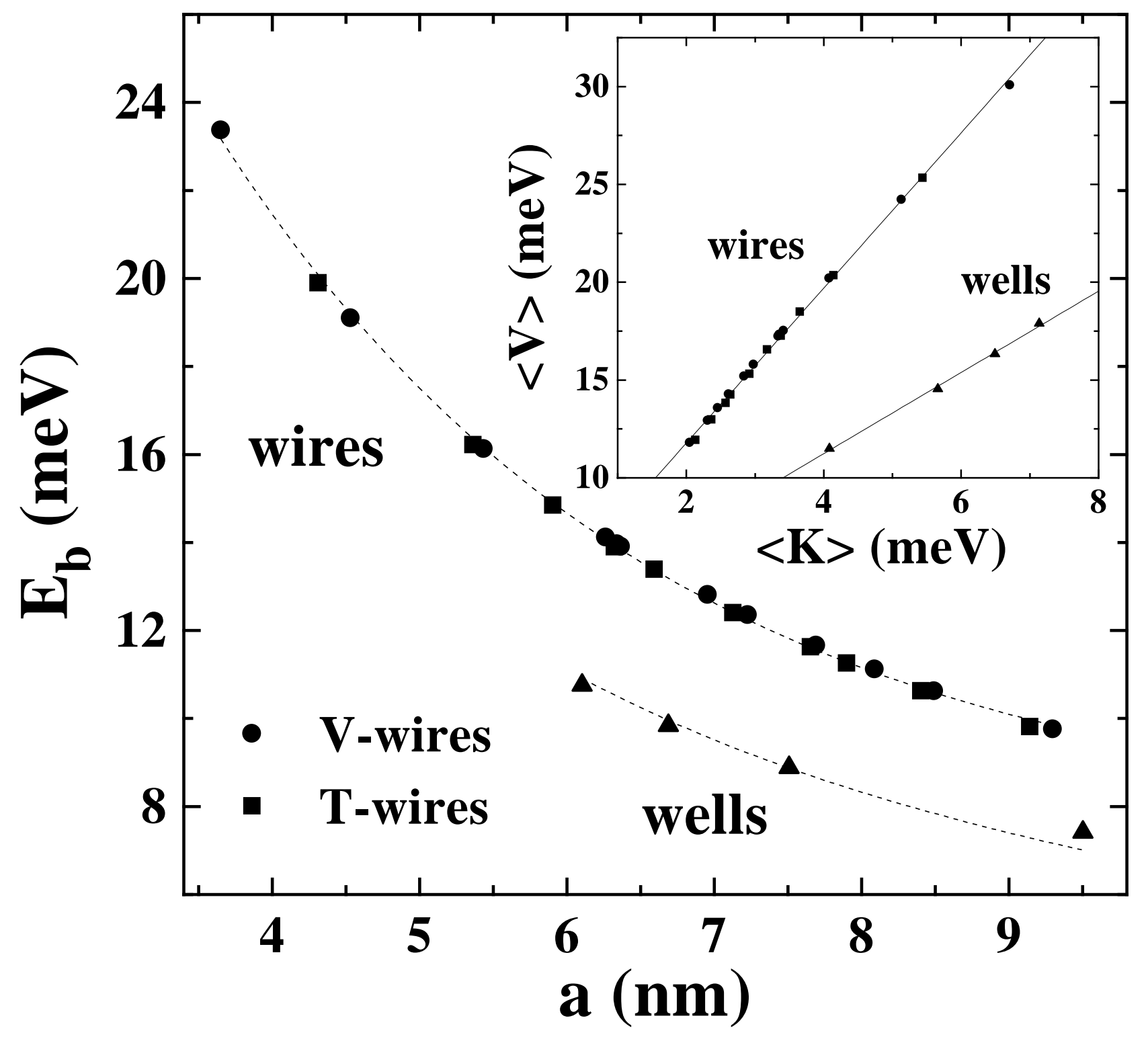

$m$
$\dot{00}$
$\dot{1}$
1
$\dot{\pi}$
0
$\ddot{0}$
$\frac{0}{0}$
$\frac{0}{0}$
0
0 